\title{
Scattering-independent anomalous Nernst effect in ferromagnets
}

\author{
Jürgen Weischenberg, ${ }^{*}$ Frank Freimuth, Stefan Blügel, and Yuriy Mokrousov \\ Peter Grünberg Institut and Institute for Advanced Simulation, Forschungszentrum Jülich and JARA, 52425 Jülich, Germany
}

(Received 30 October 2012; published 15 February 2013)

\begin{abstract}
Using the full-potential linearized augmented plane-wave method within the density functional theory, we compute all contributions to the scattering-independent part of the thermoelectric conductivity tensor $\hat{\alpha}$, namely, the intrinsic contribution $\hat{\alpha}^{\text {ic }}$ and the side-jump contribution $\hat{\alpha}^{\mathrm{sj}}$. For the ferromagnetic materials bcc Fe, hep Co, fcc $\mathrm{Ni}$, and $L 1_{0}$ ordered alloys FePd and FePt, our investigations of the energy and temperature dependence of the intrinsic and side-jump contributions show that they are both of equal importance. Overall, our calculations are able to correctly reproduce the order of magnitude and sign of the experimentally measured signal, suggesting that the scattering-independent part plays an important role in the anomalous Nernst effect of ferromagnets.
\end{abstract}

DOI: 10.1103/PhysRevB.87.060406

PACS number(s): 72.15.Jf, 71.70.Ej, 72.15.Eb, 72.25.Ba

When a thermal gradient is applied to a single-domain ferromagnetic material, the anomalous Nernst effect (ANE) shows itself as an electric field $\mathbf{E}$ that emerges in a direction perpendicular to the applied thermal gradient $-\nabla T$ and perpendicular to the sample's magnetization M. ${ }^{1}$ Prominently, the Nernst signal can be used as a probing tool for the vortex phase in type II superconductors ${ }^{2,3}$ and it has been also discussed in spinel ferromagnets ${ }^{4}$ and on a surface of a topological insulator. ${ }^{5}$ However, despite increasing interest in this phenomenon in the past years, ${ }^{6}$ attempts to predict from first principles the values of the ANE in metallic ferromagnets, such as bcc Fe, have yet to be made.

It is convenient to quantify the ANE in terms of the thermoelectric conductivity tensor $\hat{\alpha}$, also called the Peltier or Nernst conductivity tensor. In linear response theory, the expression for the charge current $\mathbf{j}$ in the presence of a weak electric field and a small thermal gradient reads ${ }^{7}$

$$
\mathbf{j}=\hat{\sigma} \cdot \mathbf{E}-\hat{\alpha} \cdot \nabla T,
$$

where the electric conductivity tensor is denoted by $\hat{\sigma}$. The matrix elements of $\hat{\alpha}$ and $\hat{\sigma}$ are related via the generalized Mott formula ${ }^{8-10}$

$$
\hat{\alpha}=-\frac{1}{e} \int d \varepsilon \frac{\partial f}{\partial \mu} \hat{\sigma} \frac{\varepsilon-\mu}{T},
$$

where $e=-|e|$ is the electronic charge, $\varepsilon$ the energy, and $\mu$ the chemical potential of the electrons which appears in the Fermi distribution function $f(\varepsilon, \mu, T)$. In ferromagnetic materials, we can replace the off-diagonal matrix elements of the conductivity tensor with the transverse anomalous Hall conductivity $\sigma^{\mathrm{AHE}},{ }^{11}$ which is usually decomposed into an intrinsic Berry curvature-driven contribution $\sigma^{\text {ic }},{ }^{12}$ and two extrinsic, stemming from disorder, contributions. Of the latter two, the first is the so-called side-jump contribution $\sigma^{\mathrm{sj}}$, which is caused by the scattering of electrons off impurities but which paradoxically does not depend on their concentration $n_{i} \cdot{ }^{13}$ The second disorder-driven contribution is the skew scattering $\sigma^{\text {sk }}$, which is inversely proportional to the impurity concentration, i.e., $\sigma^{\text {sk }} \propto 1 / n_{i} .{ }^{14,15}$ The Mott relation, Eq. (2), implies that the thermoelectric conductivity tensor can be decomposed in an analogous way:

$$
\hat{\alpha}=\hat{\alpha}^{\mathrm{ic}}+\hat{\alpha}^{\mathrm{sj}}+\hat{\alpha}^{\mathrm{sk}} \text {. }
$$

The difficulties in understanding the origins of the anomalous Hall effect (AHE) have greatly impeded the progress in the field of the ANE as well. For example, on the side of qualitative theory of solids, we are aware of only a single estimate for the value of $\hat{\alpha}^{\text {ic }}$ in the cuprate $\mathrm{CuCr}_{2} \mathrm{Se}_{4-x} \mathrm{Br}_{x}$. ${ }^{16}$ However, as we have recently shown, all scattering-independent contributions to the AHE, that is, $\hat{\sigma}^{\text {ic }}$ and $\hat{\sigma}^{\mathrm{sj}}$, can be calculated from first principles on an equal footing from the knowledge of the electronic structure of the pristine crystal alone. ${ }^{17}$ Since skew scattering is suppressed for metals outside the extremely pure regime, ${ }^{18,19}$ the calculated values for $\hat{\sigma}^{\text {ic }}$ and $\hat{\sigma}^{\mathrm{sj}}$ allow for a quantitative comparison between theory and experiment. In the present Rapid Communication, we extend this methodology to the ANE. We calculate all the scattering-independent contributions to the thermoelectric conductivity tensor, $\hat{\alpha}^{\text {ic }}$ and $\hat{\alpha}^{\mathrm{sj}}$, in bec Fe, hep Co, fcc Ni, and $L 1_{0}$ ordered alloys FePd and FePt. By comparison to experimental data, we show that $\hat{\alpha}^{\text {ic }}$ and $\hat{\alpha}^{\text {sj }}$ provide the correct order of magnitude and sign of the anomalous Nernst signal in transition-metal ferromagnets. We also make predictions concerning the temperature dependence of the scattering-independent ANE.

Our approach is based on electronic structure calculations performed within the full-potential linearized augmented plane-wave method as implemented in the Jülich density functional theory code FLEUR. ${ }^{20}$ The matrix elements of the multiband Bloch Hamiltonian $\hat{H}(\mathbf{k})$ in the basis of maximally localized Wannier functions ${ }^{21,22}$ have been computed using the Wannier interpolation technique ${ }^{23}$ and inserted into the equations for $\hat{\sigma}^{\text {ic }}$ and $\hat{\sigma}^{\text {sj }}$ obtained within the Kubo-Středa formalism, ${ }^{24}$ assuming short-range disorder in the system. ${ }^{25}$ The knowledge of any free parameters is not required in this scheme. For the evaluation of the integral in Eq. (2), we adopted an energy grid that was denser at low temperatures, since the energy derivative of the Fermi function becomes a $\delta$ distribution in this limit. Near room temperature, we found that an energy spacing of $\Delta \varepsilon \approx 5 \mathrm{meV}$ offered the best trade-off between accuracy and computational cost, leading to an error of about $2 \%$ for $\hat{\alpha}$.

It follows from an argument by Berger ${ }^{26}$ that large values for the components of $\hat{\alpha}$ could not arise if electrons of different energies experienced an AHE of the same magnitude and sign, since the transverse velocities of electrons diffusing down the applied temperature gradient would then cancel with those of the less energetic electrons diffusing up the temperature 

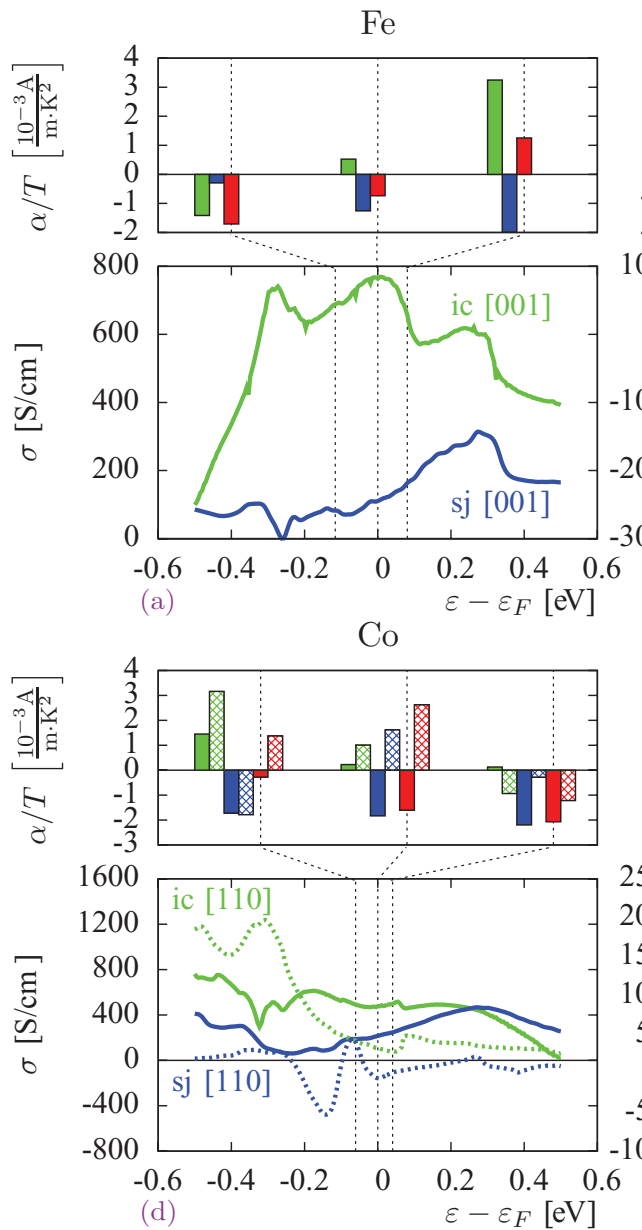
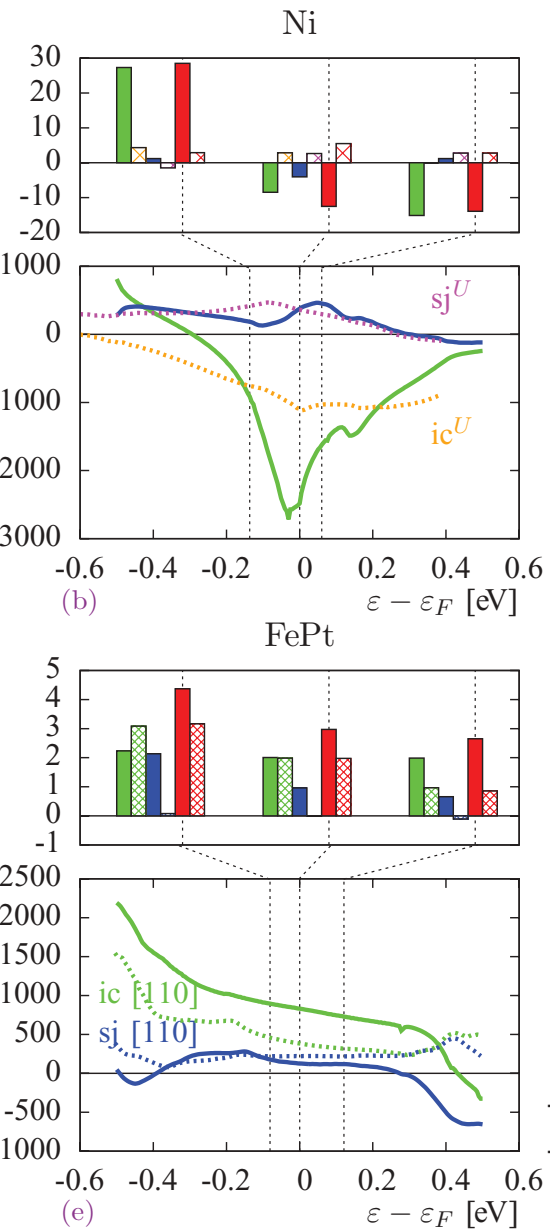

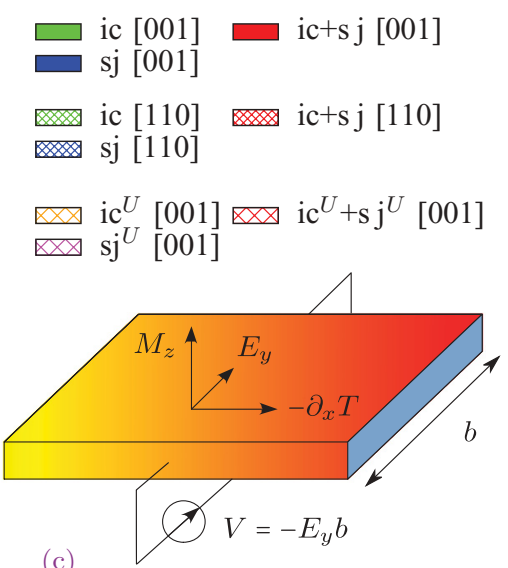

$\mathrm{FePd}$

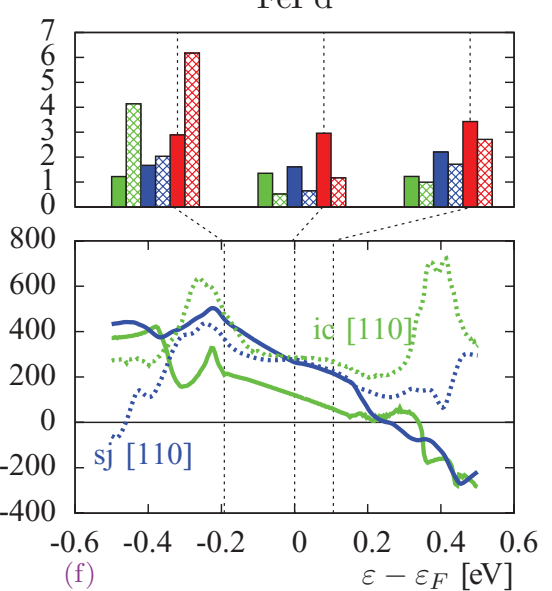

FIG. 1. (Color online) ANE at $T=300 \mathrm{~K}$ in Fe, Ni, Co, FePt, and FePd for different magnetization directions. The bar diagrams in the first row of (a), (b), (d)-(f) show the values of $\alpha=\alpha^{\text {ic }}+\alpha^{\text {sj }}$ at different energy positions. The true Fermi energy level in each material lies at the position of the middle vertical line. The line diagrams in the lower row of (a), (b), (d)-(f) depict the energy dependence of $\sigma=\sigma^{\text {ic }}+\sigma^{\text {sj }}$. In (b), hatched bars and dotted lines stand for GGA $+U$ calculations in Ni with $U=3.9 \mathrm{eV}$ and $J=1.1 \mathrm{eV}$. In (d)-(f), hatched bars and dotted lines stand for calculations in $\mathrm{Co}, \mathrm{FePt}$, and FePd with the magnetization pointing along the [110] direction. A sketch of the experimental setup is shown in (c).

gradient and the net transverse current would be zero. The same can also be deduced from the Mott formula Eq. (2): Since the product $\left(\partial_{\mu} f\right) \cdot(\varepsilon-\mu)$ is an antisymmetric function with respect to the Fermi energy level $\varepsilon_{F}=\mu$, the ANE would vanish if the AHE conductivity $\sigma^{\mathrm{AHE}}$ was a symmetric function around $\varepsilon_{F}$, i.e., if it was equal for a pair of energy values with the same distance to $\varepsilon_{F}$.

In Figs. 1(a), 1(b), and 1(d)-1(f), the component $\sigma \equiv$ $\boldsymbol{\sigma} \cdot \hat{\mathbf{M}}$ of the anomalous Hall vector $\boldsymbol{\sigma}$ and the component $\alpha \equiv \boldsymbol{\alpha} \cdot \hat{\mathbf{M}}$ of the thermoelectric conductivity vector $\boldsymbol{\alpha}$ parallel to the magnetization direction $\hat{\mathbf{M}} \equiv \mathbf{M} /|\mathbf{M}|$ are shown. They have been computed from Eq. (2) at room temperature as a function of the Fermi energy level in Fe, Ni, Co, FePt, and FePd. For the [001] magnetization direction, $\sigma$ and $\alpha$ correspond to the tensor elements $\sigma_{x y}$ and $\alpha_{x y}$, while for the [110] magnetization direction, they correspond to the tensor elements $\left(\sigma_{y z}+\sigma_{z x}\right) / \sqrt{2}$ and $\left(\alpha_{y z}+\alpha_{z x}\right) / \sqrt{2}{ }^{27}$ In bcc Fe, we observe that the intrinsic contribution $\sigma^{\text {ic }}$ is nearly symmetric around the Fermi level $\varepsilon_{F}$, resulting in a rather small value of $\alpha^{\text {ic }}$. If we shift the Fermi energy artificially by $+0.08 \mathrm{eV}$, the course of $\sigma^{\text {ic }}$ gets more asymmetric with respect to the new Fermi energy and the value of $\alpha^{\text {ic }}$ increases significantly by a factor of 6. As a consequence, the total value $\alpha^{\mathrm{ic}}+\alpha^{\mathrm{sj}}$ changes sign and becomes positive. If the Fermi energy level is lowered by $-0.12 \mathrm{eV}$ instead, all contributions become negative. In general, we find that the thermoelectric conductivity in $\mathrm{Fe}$, $\mathrm{Co}$, and $\mathrm{Ni}$ appears to be highly sensitive to the position of the Fermi level, suggesting that the ANE in ferromagnets can be easily tuned by, e.g., suitable doping. On the other hand, for the compounds FePd and FePt the Fermi energy dependence of $\alpha$ is much less pronounced.

By now it is established that the side-jump contribution to the AHE is important in FePd whereas the intrinsic AHE is dominant in FePt. ${ }^{28,29}$ As follows from Figs. 1(e) and 1(f), this statement also applies to the ANE in these materials, i.e., $\alpha^{\mathrm{sj}}\left(\varepsilon_{F}\right)$ is as large as $\alpha^{\mathrm{ic}}\left(\varepsilon_{F}\right)$ in FePd, but only half of this value in FePt. This crossover behavior is caused by the different spin-orbit interaction strength of $\mathrm{Pd}$ and $\mathrm{Pt}$ atoms. ${ }^{29,30}$ In $\mathrm{Fe}$ and $\mathrm{Co}$, the magnitude of $\alpha^{\mathrm{sj}}\left(\varepsilon_{F}\right)$ is greater than that of $\alpha^{\mathrm{ic}}\left(\varepsilon_{F}\right)$, albeit $\sigma^{\mathrm{sj}}\left(\varepsilon_{F}\right)$ being smaller than $\sigma^{\mathrm{ic}}\left(\varepsilon_{F}\right)$ in both materials. 
TABLE I. Comparison of $Q_{1}^{\mathrm{ic}+\mathrm{sj}}$ with experimental data for $Q_{1}$ in units of $10^{-11} \mathrm{~V} /(\mathrm{K} \mathrm{G})$ near room temperature. For $\mathrm{Ni}$, the values of $U$ and $J$ are in $\mathrm{eV}$.

\begin{tabular}{lrrrc}
\hline \hline & $Q_{1}^{\text {ic }}$ & \multicolumn{1}{c}{$Q_{1}^{\text {sj }}$} & $Q_{1}^{\text {ic }+ \text { sj }}$ & $Q_{1}$ expt. $^{\text {a }}$ \\
\hline $\mathrm{Fe}$ & -0.52 & -0.39 & -0.91 & -0.81 to -2.08 \\
$\mathrm{Co}[001]$ & 1.00 & 0.07 & 1.07 & 2.00 to 2.19 \\
$\quad[110]$ & 0.24 & 0.03 & 0.27 & 3.04 to 7.31 \\
$\mathrm{Ni} U=0.0, J=0.0$ & -15.12 & -1.09 & -16.20 & \\
$U=1.9, J=1.1$ & -4.40 & 1.98 & -2.42 & 5.60 \\
$U=3.9, J=1.1$ & -0.28 & 1.48 & 1.20 & 5.88 \\
$\mathrm{FePt}$ & 4.61 & 1.27 & & \\
\hline
\end{tabular}

${ }^{\mathrm{a}}$ Reference 27.

In analogy to the $\mathrm{AHE},{ }^{31}$ one might suspect that the ANE is highly anisotropic with respect to the direction of the magnetization in the crystal for hcp Co, and $L 1_{0}$ ordered FePd and FePt alloys, due to their uniaxial crystal structure. Indeed, at the Fermi energy, the side-jump contribution $\alpha^{\mathrm{sj}}$ switches its sign in Co and is distinctly reduced in FePd and FePt as the direction of the magnetization is changed from [001] to [110] direction. However, the anisotropy of the intrinsic contribution $\alpha^{\text {ic }}$ is not that strong. Such a different dependence of $\alpha^{\text {ic }}$ and $\alpha^{\mathrm{sj}}$ on the magnetization direction may be attributed to the different distribution of $\sigma^{\text {ic }}$ and $\sigma^{\text {sj }}$ in the Brillouin zone of these materials. $^{17}$

The ANE in fcc Ni presents an exceptional case, since the theoretical value of the thermoelectric conductivity in this material is much larger than in other considered compounds [see Fig. 1(b)]. In Ni, the intrinsic anomalous Hall conductivity is sharply peaked near the Fermi energy, and the respective value for the ANE depends on which side of the peak it is evaluated. There are many indications, however, that the large value of $\sigma^{\text {ic }}$ in $\mathrm{Ni}$ is mainly an artifact of the local density approximation (LDA) or the generalized gradient approximation (GGA), because correlation effects among the $3 d$ electrons in this material become of crucial importance for its properties. ${ }^{17,32,33}$ For this reason, we have adopted the same approach as in our previous work and took the correlation effects into account within the GGA $+U$ scheme. ${ }^{34}$ For the intra-atomic Coulomb repulsion and exchange parameters $U$ and $J$, we chose values up to 3.9 and $1.1 \mathrm{eV}$, respectively. This choice of parameters has been found to greatly improve the agreement of the calculated AHE in Ni. Figure 1(b) reveals that correlations have also a significant effect on the energy dependence of $\sigma^{\mathrm{AHE}}$ and $\alpha$ in Ni. We observe that the peak in the intrinsic contribution to the AHE flattens out, whereas the side-jump contribution remains mostly unaffected upon including the $U$. This can again be understood from the different behavior of the two effects at the Fermi surface. ${ }^{17}$ Upon including $U$, the intrinsic contribution $\alpha^{\text {ic }}$ and the side-jump contribution $\alpha^{\mathrm{sj}}$ change their sign and the magnitude of the thermoelectric conductivity is greatly reduced.

For comparison with experiment, we consider the situation in which a temperature gradient in the $\hat{x}$ direction, $-\partial_{x} T$, is applied to an electrically isolated sample perpendicular to the magnetization $\mathbf{M} \| \hat{z}$ [see Fig. 1(c)]. As a function of the magnetic field strength $|\mathbf{H}|=H_{z}$ and magnetization $|\mathbf{M}|=$ $M_{z}$, the Nernst effect obeys a law of the type $E_{y} /\left(-\partial_{x} T\right)=$ $H_{z} Q_{0}+4 \pi M_{z} Q_{1}$, where $Q_{0}$ and $Q_{1}$ are the ordinary and anomalous Nernst coefficients, respectively. ${ }^{35}$ However, in ferromagnetic materials, the ordinary Nernst coefficient is very small, ${ }^{36} Q_{0} \ll Q_{1}$. The remaining coefficient $Q_{1}$ is generally measured in a zero-current configuration, $j_{x}=j_{y}=$ 0 , with the boundary condition $\partial_{y} T=0 .{ }^{37}$ For a spatially uniform sample in an experimental setup as depicted in Fig. 1(c), it holds that $\sigma_{x x}=\sigma_{y y}, \sigma_{x y}=-\sigma_{y x}$, and likewise for the components of $\hat{\alpha}$. In this scenario, we obtain from Eq. (1)

$$
\begin{aligned}
4 \pi M_{z} Q_{1} & =\rho_{x x}\left(\alpha_{x y}-S \sigma_{x y}\right) \\
& =\rho_{x x}\left(\alpha_{x y}^{\mathrm{ic}}-S \sigma_{x y}^{\mathrm{ic}}\right)+\rho_{x x}\left(\alpha_{x y}^{\mathrm{sj}}-S \sigma_{x y}^{\mathrm{sj}}\right),
\end{aligned}
$$

where $\rho_{x x}=1 / \sigma_{x x}$ is the resistivity of the sample and the so-called Seebeck coefficient is defined by $S \equiv E_{x} / \partial_{x} T=$ $\alpha_{x x} / \sigma_{x x}$. The last line of Eq. (4) may be interpreted as $4 \pi M_{z}\left(Q_{1}^{\mathrm{ic}}+Q_{1}^{\mathrm{sj}}\right)$, where the intrinsic contribution to the anomalous Nernst coefficient is denoted by $Q_{1}^{\text {ic }}$ and the side-jump contribution is denoted by $Q_{1}^{\mathrm{sj}}$. While the Seebeck coefficient $S$ describes the conversion of a thermal current into a longitudinal electrical current, the Nernst coefficient $Q_{1}$ is a measure of the corresponding transverse effect. Even though the value of the temperature gradient $-\partial_{x} T$ and the strength of the magnetic induction $B_{z}$ do not appear in Eq. (4), they seem to have a great influence on the ANE experimentally. ${ }^{38,39}$ In particular, $B_{z}$ influences the magnitude of the magnetization and the corresponding electronic structure. However, values for $Q_{1}, \rho_{x x}, \alpha_{x y}, S, \sigma_{x y}$, and $M_{z}$ were not measured simultaneously in most experiments. We therefore gathered values for the resistivity $\rho_{x x}$ and Seebeck coefficient $S$ from various sources and computed the scattering-independent contribution $Q_{1}^{\mathrm{ic}+\mathrm{sj}}=Q_{1}^{\mathrm{ic}}+Q_{1}^{\mathrm{sj}}$ to the anomalous Nernst coefficient following Eq. (4) (details of this procedure can be found in the Supplemental Material). ${ }^{27}$

A comparison of our calculated values with experimental data is presented in Table I. The experimental values show a considerable spread, which reflects the fact that the ANE is found to depend sensitively on experimental details and material-specific parameters. Nevertheless, it can clearly be seen that the inclusion of the side-jump contribution is crucial for $\mathrm{Fe}$ and FePt and lets theory and experiment match very well: $Q_{1}^{\text {ic }+ \text { sj }}$ is about $112 \%$ of the smaller experimental value in $\mathrm{Fe}$ and $105 \%$ of the experimental value in FePt. For Co, the side-jump contribution to $Q_{1}^{\text {ic }+ \text { sj }}$ is relatively small and only around $10 \%$ for both magnetization directions, but it 

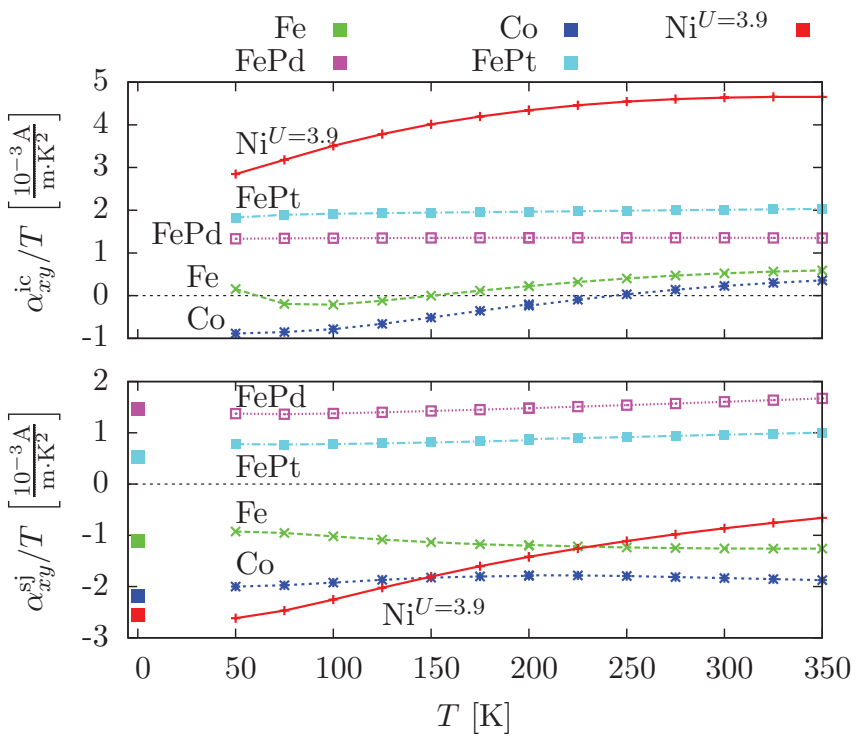

FIG. 2. (Color online) Temperature dependence of the intrinsic and side-jump contributions to the ANE. The squares at $T=0 \mathrm{~K}$ stand for the values of $\alpha_{x y}^{\mathrm{sj}} / T$ computed from Eq. (5).

still brings the theoretical value for the anomalous Nernst coefficient closer to experiment. The lack of better agreement may be due to the fact that for the Seebeck coefficient in Co solely experimental data for polycrystalline samples has been available, but the corresponding value for monocrystalline samples should be inserted into Eq. (4) instead. Remarkably, when the magnetization is changed from the [001] into the [110] direction, the sign change of $\alpha^{\mathrm{sj}}$ in Co at the Fermi energy level is compensated by the sign change of $\sigma^{\mathrm{sj}}$, and the net contribution $Q_{1}^{\mathrm{sj}}$ stays roughly the same. For Ni, the value calculated in bare GGA differs drastically from experiment, and even has the wrong sign. However, when the value of $U$ is increased within GGA $+U$, the calculated value approaches the experimental result in magnitude and sign. This suggests that the main reason for the discrepancy between experiment and theory in $\mathrm{Ni}$ originates from an inadequate description of the electronic structure in the vicinity of the Fermi energy level within GGA. ${ }^{40}$

Overall, the values in Table I demonstrate that the intrinsic and side-jump contributions play an important role in the ANE of ferromagnets. This finding is consistent with earlier studies in this field, which examined the behavior of the anomalous Nernst coefficient $Q_{1}$ as a function of the resistivity $\rho_{x x}$. As far as the scattering-independent contributions are concerned, one would expect a linear dependence of the form $Q_{1} / T \propto$ $\rho_{x x},{ }^{26}$ which is also observed in experiment. ${ }^{41-45}$ Our work substantiates these observations with quantitative analysis.
The calculated temperature dependence of the thermoelectric conductivity tensor for [001] magnetization direction is depicted in Fig. 2. $\alpha_{x y}^{\text {ic }}$ is positive at $300 \mathrm{~K}$, but changes its sign in $\mathrm{Fe}$ and $\mathrm{Co}$ as the temperature is decreased. Below $50 \mathrm{~K}$, it becomes positive again in Fe. The importance of the side-jump contribution to the ANE is stressed by the fact that for considered materials $\alpha_{x y}^{\mathrm{sj}}$ is of the same order of magnitude or even larger than $\alpha_{x y}^{\text {ic }}$. In contrast to the intrinsic contribution, $\alpha_{x y}^{\mathrm{sj}}$ does not change its sign. While the temperature dependence of $\alpha_{x y}^{\mathrm{sj}} / T$ is almost absent in FePd and FePt, it ranges from $-2.6 \times 10^{-3} \mathrm{~A} /\left(\mathrm{m} \mathrm{K}^{2}\right)$ at $T=50 \mathrm{~K}$ to $-0.7 \times 10^{-3} \mathrm{~A} /\left(\mathrm{m} \mathrm{K}^{2}\right)$ at $T=350 \mathrm{~K}$ in Ni.

In the zero temperature limit, one can apply the Sommerfeld expansion to the integral in Eq. (2) to obtain the standard Mott formula which relates the ANE to the energy derivative of the AHE: ${ }^{46}$

$$
\frac{\alpha_{x y}}{T}=-\frac{\pi^{2} k_{B}^{2}}{3 e}\left[\frac{d \sigma_{x y}}{d \varepsilon}\right]_{\varepsilon=\varepsilon_{F}} .
$$

For the intrinsic Nernst effect, it is known that the above formula may be violated as $T \rightarrow 0 \mathrm{~K} .{ }^{6}$ Indeed, we find that the energy derivative of the intrinsic contribution converges only very slowly with respect to the number of $\mathbf{k}$ points in the Brillouin zone that are used for the evaluation of $\sigma^{\text {ic }}$. The slow convergence is due to the sensitivity of the Berry curvature to the position of the Fermi energy, especially when the latter approaches avoided band crossings or points of band degeneracy. ${ }^{47}$ However, for the side-jump contribution, Mott's formula Eq. (5) holds, as can be seen by interpolating the curves in Fig. 2 to $T=0 \mathrm{~K}$. Apart from Ni, it yields a rather good estimate for the value of $\alpha_{x y}^{\mathrm{sj}} / T$ at room temperature as well.

In summary, we presented the $a b$ initio calculations of the scattering-independent contributions to the ANE in several ferromagnets. The theoretical values for the thermoelectric conductivity tensor and the comparison of the calculated anomalous Nernst coefficient with experiment suggests that the ANE in elementary $\mathrm{Fe}, \mathrm{Co}, \mathrm{Ni}$, in the ferromagnetic alloy $\mathrm{FePt}$, and presumably also in FePd is largely caused by the intrinsic and side-jump mechanisms. Discrepancies between theory and experiment in $\mathrm{Ni}$ are likely due to the imprecise description of correlation effects within bare GGA, which can be remedied by GGA $+U$ calculations.

We thank J. Sinova for fruitful discussions and gratefully acknowledge Jülich Supercomputing Centre for computing time as well as funding by the HGF-YIG programme VH-NG513. J.W. was supported under Grant No. SPP 1538 SpinCaT by the German Science Foundation.
*Corresponding author: j.weischenberg@fz-juelich.de

${ }^{1}$ W. Nernst, Ann. Phys. 267, 760 (1887).

${ }^{2}$ Z. A. Xu, N. P. Ong, Y. Wang, T. Kakeshita, and S. Uchida, Nature (London) 406, 486 (2000).

${ }^{3}$ Y. Wang, Z. A. Xu, T. Kakeshita, S. Uchida, S. Ono, Y. Ando, and N. P. Ong, Phys. Rev. B 64, 224519 (2001).
${ }^{4}$ W.-L. Lee, S. Watauchi, V. L. Miller, R. J. Cava, and N. P. Ong, Phys. Rev. Lett. 93, 226601 (2004).

${ }^{5}$ T. Yokoyama and S. Murakami, Phys. Rev. B 83, 161407 (2011).

${ }^{6}$ D. L. Bergman and V. Oganesyan, Phys. Rev. Lett. 104, 066601 (2010).

${ }^{7}$ J. M. Luttinger, Phys. Rev. 135, A1505 (1964). 
${ }^{8}$ L. Smřcka and P. Středa, J. Phys. C 10, 2153 (1977).

${ }^{9}$ M. Jonson and G. D. Mahan, Phys. Rev. B 21, 4223 (1980).

${ }^{10}$ M. J. Kearney and P. N. Butcher, J. Phys. C 21, L265 (1988).

${ }^{11}$ E. M. Pugh, Phys. Rev. 36, 1503 (1930).

${ }^{12}$ R. Karplus and J. M. Luttinger, Phys. Rev. 95, 1154 (1954).

${ }^{13}$ L. Berger, Phys. Rev. B 2, 4559 (1970).

${ }^{14}$ J. Smit, Physica 21, 877 (1955).

${ }^{15}$ J. Smit, Physica 24, 39 (1958).

${ }^{16}$ D. Xiao, Y. Yao, Z. Fang, and Q. Niu, Phys. Rev. Lett. 97, 026603 (2006).

${ }^{17}$ J. Weischenberg, F. Freimuth, J. Sinova, S. Blügel, and Y. Mokrousov, Phys. Rev. Lett. 107, 106601 (2011).

${ }^{18}$ T. Miyasato, N. Abe, T. Fujii, A. Asamitsu, S. Onoda, Y. Onose, N. Nagaosa, and Y. Tokura, Phys. Rev. Lett. 99, 086602 (2007).

${ }^{19}$ N. Nagaosa, J. Sinova, S. Onoda, A. H. MacDonald, and N. P. Ong, Rev. Mod. Phys. 82, 1539 (2010).

${ }^{20}$ FLEUR web page, http://www.flapw.de

${ }^{21}$ F. Freimuth, Y. Mokrousov, D. Wortmann, S. Heinze, and S. Blügel, Phys. Rev. B 78, 035120 (2008).

${ }^{22}$ A. A. Mostofi, J. R. Yates, Y.-S. Lee, I. Souza, D. Vanderbilt, and N. Marzari, Comput. Phys. Commun. 178, 685 (2008).

${ }^{23}$ J. R. Yates, X. Wang, D. Vanderbilt, and I. Souza, Phys. Rev. B 75, 195121 (2007).

${ }^{24}$ P. Středa and L. Smrčka, Phys. Status Solidi B 70, 537 (1975).

${ }^{25}$ A. A. Kovalev, J. Sinova, and Y. Tserkovnyak, Phys. Rev. Lett. 105, 036601 (2010).

${ }^{26}$ L. Berger, Phys. Rev. B 5, 1862 (1972)

${ }^{27}$ See Supplemental Material at http://link.aps.org/supplemental/ 10.1103/PhysRevB.87.060406 for further information.

${ }^{28}$ P. He, L. Ma, Z. Shi, G. Y. Guo, J.-G. Zheng, Y. Xin, and S. M. Zhou, Phys. Rev. Lett. 109, 066402 (2012).
${ }^{29}$ K. M. Seemann, Y. Mokrousov, A. Aziz, J. Miguel, F. Kronast, W. Kuch, M. G. Blamire, A. T. Hindmarch, B. J. Hickey, I. Souza, and C. H. Marrows, Phys. Rev. Lett. 104, 076402 (2010).

${ }^{30}$ H. Zhang, F. Freimuth, S. Blügel, Y. Mokrousov, and I. Souza, Phys. Rev. Lett. 106, 117202 (2011).

${ }^{31}$ H. Zhang, S. Blügel, and Y. Mokrousov, Phys. Rev. B 84, 024401 (2011).

${ }^{32}$ I. Yang, S. Y. Savrasov, and G. Kotliar, Phys. Rev. Lett. 87, 216405 (2001).

${ }^{33}$ H.-R. Fuh and G.-Y. Guo, Phys. Rev. B 84, 144427 (2011).

${ }^{34}$ A. B. Shick, A. I. Liechtenstein, and W. E. Pickett, Phys. Rev. B 60, 10763 (1999).

${ }^{35}$ J.-P. Jan, in Solid State Physics, edited by F. Seitz and D. Turnbull (Academic, New York, 1957), Vol. 5, pp. 1-96.

${ }^{36}$ G. S. Nielsen, Philos. Mag. 18, 575 (1934).

${ }^{37}$ E. H. Butler and E. M. Pugh, Phys. Rev. 57, 916 (1940).

${ }^{38}$ L. L. Campbell, Phys. Rev. 26, 416 (1908).

${ }^{39}$ L. L. Campbell, Galvanomagnetic and Thermomagnetic Effects (Longmans, Green and Co., London, 1923).

${ }^{40}$ Further reasons for the discrepancy might be the temperature dependence of the electronic structure or details of dynamical Coulomb screening of $d$ electrons in $\mathrm{Ni}$, etc.

${ }^{41}$ I. Campbell, J. Magn. Magn. Mater. 12, 31 (1979).

${ }^{42}$ T. Luciński and J. Baszyński, J. Magn. Magn. Mater. 127, 57 (1993).

${ }^{43}$ E. I. Kondorskii and R. P. Vasileva, Sov. Phys. JETP 18, 277 (1964).

${ }^{44}$ A. Cheremushkina and R. P. Vasileva, Sov. Phys. Solid State 8, 659 (1966).

${ }^{45}$ R. P. Vasil'eva and B. Akmuradov, Russ. Phys. J. 15, 814 (1972).

${ }^{46}$ N. W. Ashcroft and D. N. Mermin, Solid State Physics (Saunders College Publishing, Philadelphia, 1988).

${ }^{47}$ Y. Yao, L. Kleinman, A. H. MacDonald, J. Sinova, T. Jungwirth, D.-s. Wang, E. Wang, and Q. Niu, Phys. Rev. Lett. 92, 037204 (2004). 